\title{
Early clinical outcomes after transapical aortic valve implantation: A propensity-matched comparison with conventional aortic valve replacement
}

\author{
Jennifer Higgins, MHSc, MD, ${ }^{\mathrm{a}}$ Jian Ye, MD, ${ }^{\mathrm{a}}$ Karin H. Humphries, DSc, ${ }^{\text {a,b }}$ Anson Cheung, MD, \\ David A. Wood, MD, ${ }^{b}$ John G. Webb, MD, ${ }^{b}$ and Samuel V. Lichtenstein, MD ${ }^{a}$
}

\begin{abstract}
Objective: Aortic valve replacement remains the standard treatment for symptomatic severe aortic stenosis. However, catheter-based approaches have recently emerged as therapeutic options for high-risk surgical candidates. The objective of this study is to use propensity scoring to compare early clinical outcomes after transapical aortic valve implantation and conventional aortic valve replacement.
\end{abstract}

\begin{abstract}
Method: Propensity scoring based on logistic regression modeling of 16 preoperative patient characteristics was used to identify a group of very high-risk patients undergoing isolated conventional aortic valve replacement comparable to those patients undergoing transapical aortic valve implantation. McNemar's test was used to compare early clinical outcomes between the 2 treatment groups, including 30-day mortality and in-hospital postoperative complications.
\end{abstract}

\begin{abstract}
Results: Ninety-two patients receiving transapical aortic valve implantation between October 2005 and April 2010 met inclusion criteria for this study. Half of these patients were successfully matched 1:1 to a patient receiving conventional aortic valve replacement. Baseline characteristics were similar between the 2 treatment groups after propensity matching. There were 4 perioperative deaths $(8.7 \%)$ in the conventional aortic valve replacement group and 6 perioperative deaths $(13 \%)$ in the transapical aortic valve implantation group $(P>.05)$. There were no significant differences in the rates of cerebrovascular accidents, wound infections, reoperation for bleeding, or length of postoperative hospital stay between the 2 groups $(P>.05)$.

Conclusions: Among high-risk propensity-matched patients, early clinical outcomes are similar after transapical aortic valve implantation and conventional aortic valve replacement. However, given the likelihood of residual selection bias, a prospective randomized trial is necessary to adequately compare the clinical outcomes after these 2 aortic valve procedures. (J Thorac Cardiovasc Surg 2011;142:e47-52)
\end{abstract}

Aortic valve replacement (AVR) is the treatment of choice for severe symptomatic aortic stenosis. ${ }^{1}$ However, less invasive catheter-based approaches, including transarterial and transapical aortic valve implantation (AVI), have emerged over the past few years as treatment options for those with significant comorbidities. ${ }^{2-8}$ The PARTNER trial recently demonstrated that in patients with severe aortic stenosis who were not suitable candidates for surgery, transfemoral AVI, compared with medical management, significantly reduced the risk of death from any cause, the composite end point of death from any cause/repeat hospitalization, and cardiac symptoms, despite the higher incidence of major strokes and major vascular events. ${ }^{9}$

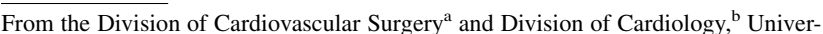
sity of British Columbia, Vancouver, British Columbia, Canada.

Disclosures: Authors have nothing to disclose with regard to commercial support.

Received for publication Nov 21, 2010; revisions received Jan 16, 2011; accepted for publication Feb 9, 2011; available ahead of print May 18, 2011.

Address for reprints: Jian Ye, MD, Division of Cardiovascular Surgery, St Paul's Hospital, 1081 Burrard Street, Vancouver, BC, V6Z 1Y6, Canada (E-mail: jye@ providencehealth.bc.ca).

$0022-5223 / \$ 36.00$

Copyright (c) 2011 by The American Association for Thoracic Surgery doi:10.1016/j.jtcvs.2011.02.045
}

Transapical AVI is often reserved for individuals with poor peripheral arterial access, which limits the transarterial femoral approach. ${ }^{3,6}$ Initial experience with transapical AVI, in carefully selected high-risk patients, has been favorable with intraoperative mortality of $0 \%$ to $2.6 \%, 30$-day mortality of $4.3 \%$ to $23 \%$, and 1 -year survival of $71.4 \%$ to $85 \%{ }^{2,3,6-8,10-14}$ In Vancouver, among our initial 71 patients, overall survivals at 24 and 36 months were $66.3 \% \pm 6.4 \%$ and $58.0 \% \pm 9.5 \%$, respectively. ${ }^{15}$ Among 59 patients who survived at least 30 days, 24 - and 36-month survivals were $79.8 \% \pm 6.4 \%$ and $69.8 \% \pm 10.9 \%$, respectively. ${ }^{15}$ Despite the promising early results demonstrated in these case series, it is unclear how postoperative outcomes, including survival, compare with conventional AVR, the currently accepted standard of care.

The primary difficulty in comparing clinical outcomes after these 2 aortic valve procedures is the associated selection bias. Transapical AVI is currently limited to compassionate use for individuals at extremely high risk for conventional surgery, and therefore cannot be compared with all patients undergoing conventional AVR. The objective of this study was to use propensity scoring to identify a high-risk population of patients undergoing isolated conventional AVR 


\section{Abbreviations and Acronyms \\ $\mathrm{AVI}=$ aortic valve implantation \\ $\mathrm{AVR}=$ aortic valve replacement \\ $\mathrm{CVA}=$ cerebrovascular accident \\ $\mathrm{PVD}=$ peripheral vascular disease}

comparable to the population undergoing transapical AVI, and compare clinical outcomes after the 2 procedures. Specifically, this study explored 30-day mortality, in-hospital complications, and length of hospital stay.

\section{MATERIALS AND METHODS}

Perioperative data are collected prospectively on all patients undergoing cardiac surgery in the province of British Columbia and maintained in the Cardiac Services BC registry. This database was used to identify 2 groups of patients: (1) patients undergoing transapical AVI and (2) patients undergoing conventional AVR. Conventional AVR cases were performed in 4 hospitals in British Columbia, and transapical AVI cases were performed in a single center. These 2 groups were then subjected to inclusion and exclusion criteria. Patients were included if they received a primary isolated aortic valve procedure for severe aortic stenosis between January 2000 and April 2010. Patients were excluded if they underwent any concomitant cardiac surgery procedure; had previous aortic valve surgery; received aortic valve surgery for non-aortic stenosis, such as pure aortic insufficiency, endocarditis, aortic root aneurysm, or aortic dissection; underwent surgery before the year 2000; and had a porcelain aorta. Preoperative baseline characteristics were compared for the 2 treatment groups with the $z$ test for dichotomous variables and the unpaired Student $t$ test for continuous variables.

Propensity scoring was then used to identify a group of very high-risk patients undergoing conventional AVR comparable to those patients undergoing transapical AVI in an attempt to account for selection bias. The propensity scoring model was composed of a logistic regression model including preoperative patient characteristics that demonstrated a statistically significant imbalance between the 2 treatment groups. This large number of covariates can be included in the propensity score model because it is not subject to the limitation of parsimony that characterizes conventional logistic regression modelling. The Greedy algorithm, with a calliper of 0.001 to 0.1 , was then used to match the conventional AVR cases to the transapical AVI cases 1:1 on the basis of propensity scores. Transapical AVI cases unable to be matched were excluded from further analysis. Baseline characteristics were again compared for the 2 groups after the propensity score-based match using McNemar's test for dichotomous variables and paired Student $t$ test for continuous variables.

Finally, McNemar's test was used to compare the dichotomous clinical outcomes between the 2 treatment groups, including 30-day mortality and in-hospital postoperative complications. The in-hospital complications of interest were cerebrovascular accidents (CVAs), wound infections, and reoperation for bleeding. The Wilcoxon signed-rank test was used to compare length of postoperative hospital stay. The provincial database was reviewed to determine rates of reoperation for bleeding, and hospital discharge forms were reviewed to determine rates of CVA and wound infection and length of hospital stay.

\section{RESULTS}

\section{Patient Characteristics}

From January 2000 to April 2010, 2180 patients meeting the inclusion and exclusion criteria of this study underwent either conventional AVR $(\mathrm{n}=2088)$ or transapical AVI $(\mathrm{n}=92)$ for severe aortic stenosis in British Columbia. Patients undergoing transapical AVI were older $(81 \pm 12$ years vs $69 \pm 12$ years, $P<.0001$ ), with a smaller body surface area and a significantly increased prevalence of comorbidities, including pulmonary hypertension, peripheral vascular disease (PVD), anemia, and renal dysfunction (Table 1). Patients undergoing transapical AVI were also more likely to be female ( $63 \%$ vs $44 \%$ female, $P<.0001)$.

Sixteen preoperative variables were included in the propensity score logistic regression model (Table 2). Several measured preoperative risk factors were not used in the model because they were not independently associated with the decision to perform transapical AVI. Excluded variables included history of malignancy, hypertension, diabetes, and known liver disease. On the basis of this propensity model, 46 of the 92 patients receiving transapical AVI were successfully matched to a patient receiving conventional AVR. After matching, there were no significant differences in any of the preoperative variables between the transapical AVI and the conventional AVR groups, including those variables not used in the logistic regression model (Table 3).

Patients undergoing transapical AVI who were unable to be matched to the conventional AVR group were significantly different from those patients who were matched (Table 4). Unmatched patients were older $(83 \pm 5$ years vs $78 \pm 8$ years, $P<.001$ ), with an increased prevalence of pulmonary hypertension, PVD, anemia, and renal dysfunction $(P<.001)$ compared with the matched patients undergoing transapical AVI. Similarly, there were significant differences between matched and unmatched patients undergoing conventional AVR (Table 5).

\section{Clinical Outcomes: Propensity-Matched Groups}

Within the conventional AVR group, there were 4 perioperative deaths, whereas there were 6 perioperative deaths among the transapical AVI group (Table 6). According to McNemar's test, there was no significant difference between the two 30-day mortality rates $(P=.53)$. Among the 6 perioperative deaths in the transapical AVI group, 3 occurred among the first 15 cases performed at our center. The causes of the mortality in the transapical AVI cases are listed in Table 7. One patient experienced a cardiac arrest before valve insertion and died intraoperatively despite emergency femoral-femoral cardiopulmonary bypass.

Similarly, there were no significant differences in the rates of in-hospital complications, including CVA, wound infection, and reoperation for bleeding (Table 6). There were no perioperative CVAs or wound infections observed among the matched transapical AVI cases. Finally, there was no significant difference in the length of stay in hospital between the matched transapical AVI group and the matched conventional AVR group. 
TABLE 1. Baseline characteristics for all transapical aortic valve implantations and conventional aortic valve replacements

\begin{tabular}{|c|c|c|c|}
\hline & TAVI $(\mathbf{n}=92)$ & $\operatorname{AVR}(\mathbf{n}=2088)$ & $P$ value \\
\hline Year of surgery & 2005-2010 & 2000-2009 & \\
\hline Age (y) & $81(12)$ & $69(12)$ & $<.0001$ \\
\hline Male & $34(37 \%)$ & $1179(56 \%)$ & . 0001 \\
\hline BSA & $1.72(0.21)$ & $1.92(0.26)$ & $<.0001$ \\
\hline NYHA III/IV & $88(96 \%)$ & $864(41 \%)$ & $<.0001$ \\
\hline CCS III/IV & $37(40 \%)$ & $403(19 \%)$ & $<.0001$ \\
\hline LVEF < 35\% & $6(7 \%)$ & $117(6 \%)$ & .354 \\
\hline HTN & $85(92 \%)$ & $1359(65 \%)$ & $<.0001$ \\
\hline PHTN & $69(75 \%)$ & $302(14 \%)$ & $<.0001$ \\
\hline Dyslipidemia & $75(82 \%)$ & $985(47 \%)$ & $<.0001$ \\
\hline PVD & $68(74 \%)$ & $217(10 \%)$ & $<.0001$ \\
\hline Arrhythmia & $49(53 \%)$ & $277(13 \%)$ & $<.0001$ \\
\hline COPD & $47(51 \%)$ & $407(19 \%)$ & $<.0001$ \\
\hline CVA & $29(32 \%)$ & $407(19 \%)$ & $<.0001$ \\
\hline Renal dysfunction & $52(57 \%)$ & $310(15 \%)$ & $<.0001$ \\
\hline Diabetes & $31(34 \%)$ & $404(19 \%)$ & .0004 \\
\hline Liver disease & $18(20 \%)$ & $163(8 \%)$ & $<.0001$ \\
\hline Malignancy & $21(23 \%)$ & $301(14 \%)$ & .0130 \\
\hline Anemia & $65(71 \%)$ & $231(12 \%)$ & $<.0001$ \\
\hline PCI & $32(35 \%)$ & $121(6 \%)$ & $<.0001$ \\
\hline MI & $51(55 \%)$ & $205(10 \%)$ & $<.0001$ \\
\hline GI bleed & $19(21 \%)$ & $146(7 \%)$ & $<.0001$ \\
\hline Remote smoker & $56(61 \%)$ & $549(26 \%)$ & $<.0001$ \\
\hline Current smoker & $4(4 \%)$ & $158(8 \%)$ & .875 \\
\hline Previous sternotomy & $41(45 \%)$ & $113(5 \%)$ & $<.0001$ \\
\hline Creatinine & $124(79)$ & $94(55)$ & .0006 \\
\hline Hemoglobin & 119 (14) & $134(16)$ & $<.0001$ \\
\hline
\end{tabular}

Values expressed as mean (standard deviation) or number (\%). BSA, Body surface area; $C C S$, Canadian Cardiovascular Society; $C O P D$, chronic obstructive pulmonary disease; $C V A$, cerebrovascular accident; $G I$, gastrointestinal; HTN, hypertension; $L V E F$, left ventricular ejection fraction; $M I$, myocardial infarction; NYHA, New York Heart Association classification; $P C I$, percutaneous coronary intervention; $P H T N$, pulmonary hypertension; $P V D$, peripheral vascular disease.

\section{Clinical Outcomes: Unmatched Groups}

Relative to the matched transapical AVI group, the incidence of reoperation for bleeding was significantly higher in the unmatched group $(11 \%$ vs $15 \%, P<.001)$ (Table $8)$. However, perioperative mortality seemed to be higher in the matched transapical AVI group (13\%) compared with the unmatched group (11\%), although this difference was not statistically significant $(P=.064)$ (Table 8$)$. Review of the conventional AVR cases demonstrated an increased rate of 30 -day mortality $(9 \%$ vs $2 \%, P<.001)$ and a trend toward an increased incidence of CVA ( $4 \%$ vs $1 \%, P=.080)$ among matched AVR cases compared with unmatched cases (Table 9).

\section{DISCUSSION}

The demand for minimally invasive approaches to surgical procedures continues to increase. Over the past few years, there has been a significant expansion in the use of catheter-based approaches to manage aortic valve disease.
TABLE 2. Maximum likelihood estimates for propensity logistic regression model

\begin{tabular}{lcccr}
\hline \multicolumn{1}{c}{ Parameter } & $d f$ & Estimate & SE & \multicolumn{1}{c}{$\boldsymbol{P}>\boldsymbol{\chi}^{2}$} \\
\hline Intercept & 1 & -10.7952 & 2.8984 & .0002 \\
Age $(10$ y) & 1 & 1.1619 & 0.2771 & $<.0001$ \\
Sex & 1 & -1.0509 & 0.4491 & .0193 \\
BSA & 1 & -3.3287 & 0.9344 & .0004 \\
NYHA III/IV & 1 & 1.5298 & 0.5883 & .0093 \\
LVEF $<35 \%$ & 1 & -1.6551 & 0.7246 & .0224 \\
Previous sternotomy & 1 & 1.3357 & 0.4539 & .0033 \\
Prior CABG & 1 & 2.1248 & 0.4277 & $<.0001$ \\
PHTN & 1 & 1.2348 & 0.3832 & .0013 \\
Arrhythmia & 1 & 0.1031 & 0.3898 & .7914 \\
PVD & 1 & 1.653 & 0.3691 & $<.0001$ \\
COPD & 1 & 0.7196 & 0.3722 & .0532 \\
CVA & 1 & 0.1805 & 0.4285 & .6736 \\
Renal dysfunction & 1 & 0.1168 & 0.391 & .7652 \\
GI bleed & 1 & -0.00075 & 0.4837 & .9988 \\
Anemia & 1 & 1.7853 & 0.3844 & $<.0001$ \\
Smoker & 1 & 1.4905 & 0.3831 & $<.0001$ \\
\hline
\end{tabular}

$S E$, Standard error; $B S A$, body surface area; $C A B G$, coronary artery bypass grafting $C O P D$, chronic obstructive pulmonary disease; $C V A$, cerebrovascular accident; $P H T N$, pulmonary hypertension; $L V E F$, left ventricular ejection fraction; NYHA, New York Heart Association classification; $P V D$, peripheral vascular disease; $G I$ gastrointestinal.

These techniques are currently reserved for compassionate use among very high-risk patients, but there is considerable interest in broadening the selection criteria to include individuals who would be expected to have excellent clinical outcomes with conventional AVR. However, before these catheter-based approaches can be routinely offered to low to moderate risk or young patients, it is imperative to compare the clinical outcomes after these less invasive procedures with the outcomes achievable with the currently accepted standard of care, conventional AVR.

In comparing morbidity and mortality after transapical AVI and conventional AVR, selection bias must be addressed. Walther and colleagues ${ }^{13}$ recently used propensity scoring based on 21 preoperative variables to match $100 \%$ of 100 consecutive patients undergoing transapical AVI. However, their matching technique was inadequately described in their article. Furthermore, the successful match of all patients undergoing transapical AVI raises concerns about their selection process for transapical AVI cases and whether patients receiving this new procedure are truly any different from those receiving conventional AVR. In our study, we have clearly demonstrated that there are significant differences between the 2 treatment groups (Table 1), as would be expected given the current indications for transapical AVI.

The objective of our study was to be transparent about our matching process to accurately compare clinical outcomes between these 2 treatment groups. Despite the documented selection bias, we were able to match half of the transapical AVI cases meeting our inclusion and exclusion criteria to 
TABLE 3. Baseline characteristics for propensity-matched transapical aortic valve implantation and conventional aortic valve replacement cases

\begin{tabular}{|c|c|c|c|}
\hline & TAVI $(n=46)$ & $\operatorname{AVR}(n=46)$ & $P$ value \\
\hline Year of surgery & 2005-2010 & 2001-2009 & \\
\hline Age (y) & $78(8)$ & $78(6)$ & .996 \\
\hline Male & $20(0.43)$ & $19(0.41)$ & .700 \\
\hline BSA & $1.75(0.23)$ & $1.72(0.24)$ & .928 \\
\hline NYHA III/IV & $42(91 \%)$ & $43(93 \%)$ & 1.000 \\
\hline CCS III/IV & $15(33 \%)$ & $16(35 \%)$ & 1.000 \\
\hline $\mathrm{LVEF}<35 \%$ & $3(7 \%)$ & $6(13 \%)$ & .683 \\
\hline $\mathrm{HTN}$ & $40(87 \%)$ & $38(83 \%)$ & .789 \\
\hline PHTN & $26(57 \%)$ & $29(63 \%)$ & .689 \\
\hline Dyslipidemia & $36(78 \%)$ & $32(69 \%)$ & .522 \\
\hline PVD & $27(58 \%)$ & $25(54 \%)$ & .819 \\
\hline Arrhythmia & $21(46 \%)$ & $18(39 \%)$ & .628 \\
\hline COPD & $20(43 \%)$ & $18(39 \%)$ & .838 \\
\hline CVA & $13(28 \%)$ & $15(33 \%)$ & .814 \\
\hline Renal dysfunction & $18(39 \%)$ & $19(41 \%)$ & 1.000 \\
\hline Diabetes & $17(37 \%)$ & $14(30 \%)$ & .677 \\
\hline Liver disease & $10(22 \%)$ & $5(11 \%)$ & .267 \\
\hline Malignancy & $7(15 \%)$ & $5(11 \%)$ & .752 \\
\hline Anemia & $25(54 \%)$ & $31(67 \%)$ & .269 \\
\hline PCI & $13(28 \%)$ & $11(24 \%)$ & .789 \\
\hline MI & $22(48 \%)$ & $19(41 \%)$ & .689 \\
\hline GI bleed & $8(17 \%)$ & $8(17 \%)$ & .803 \\
\hline Remote smoker & $23(50 \%)$ & $19(41 \%)$ & .453 \\
\hline Current smoker & $3(7 \%)$ & $3(7 \%)$ & .683 \\
\hline Previous sternotomy & $17(37 \%)$ & $17(37 \%)$ & .831 \\
\hline Creatinine & $122(87)$ & $118(105)$ & .982 \\
\hline Hemoglobin & $121(15)$ & $116(15)$ & .844 \\
\hline
\end{tabular}

Values expressed as mean (standard deviation) or number (\%). TAVI, Transapical aortic valve implantation; $A V R$, aortic valve replacement; $B S A$, body surface area; $C C S$, Canadian Cardiovascular Society; COPD, chronic obstructive pulmonary disease; $C V A$, cerebrovascular accident; $H T N$, hypertension; $L V E F$, left ventricular ejection fraction; $M I$, myocardial infarction; NYHA, New York Heart Association classification; $P C I$, percutaneous coronary intervention; $P H T N$, pulmonary hypertension; $P V D$, peripheral vascular disease; $G I$, gastrointestinal.

patients receiving conventional AVR by using a propensity model that included 16 preoperative characteristics. After the match, there were no significant differences between the 2 patient samples included in the analysis. However, there were significant differences between those patients included in the match and those who could not be matched. Essentially, the lower risk transapical AVI cases were propensity matched to the highest risk conventional AVR cases. Therefore, it is imperative that the results of this study should not be extrapolated to the entire transapical AVI population. Conversely, this study does help illuminate the procedural risks for high-risk patients who may be considered for both conventional AVR and transapical AVI.

Currently at out center, patients must be turned down for conventional AVR by cardiac surgeons before they can be considered for transapical AVI. However, not all referred patients are accepted for transapical AVI. Appropriate candidates would have a life expectancy of at least 1 year, as well as a reasonable quality of life, if not for their aortic
TABLE 4. Preoperative characteristics for matched and unmatched transapical aortic valve implantation cases

\begin{tabular}{|c|c|c|c|}
\hline & $\begin{array}{l}\text { Matched TAVI } \\
\quad(n=46)\end{array}$ & $\begin{array}{c}\text { Unmatched TAVI } \\
(n=46)\end{array}$ & $P$ value \\
\hline Year of surgery & $2005-2010$ & 2006-2010 & \\
\hline Age (y) & $78(8)$ & $83(5)$ & $<.001$ \\
\hline Male & $20(43 \%)$ & $14(34 \%)$ & .371 \\
\hline BSA & $1.75(0.23)$ & $1.69(0.19)$ & .173 \\
\hline NYHA III/IV & $42(91 \%)$ & $46(100 \%)$ & .034 \\
\hline CCS III/IV & $15(33 \%)$ & $22(48 \%)$ & .139 \\
\hline LVEF $<35 \%$ & $3(7 \%)$ & $3(7 \%)$ & 1.000 \\
\hline HTN & $40(87 \%)$ & $45(98 \%)$ & .046 \\
\hline PHTN & $26(57 \%)$ & $43(93 \%)$ & $<.001$ \\
\hline Dyslipidemia & $36(78 \%)$ & $39(85 \%)$ & .387 \\
\hline PVD & $27(58 \%)$ & $41(89 \%)$ & $<.001$ \\
\hline Arrhythmia & $21(46 \%)$ & $28(60 \%)$ & .178 \\
\hline COPD & $20(43 \%)$ & $27(59 \%)$ & .124 \\
\hline CVA & $13(28 \%)$ & $16(35 \%)$ & .470 \\
\hline Renal dysfunction & $18(39 \%)$ & $34(74 \%)$ & $<.001$ \\
\hline Diabetes & $17(37 \%)$ & $14(30 \%)$ & .478 \\
\hline Liver disease & $10(22 \%)$ & $8(17 \%)$ & .546 \\
\hline Malignancy & $7(15 \%)$ & $14(30 \%)$ & .087 \\
\hline Anemia & $25(54 \%)$ & $40(87 \%)$ & $<.001$ \\
\hline PCI & $13(28 \%)$ & $19(41 \%)$ & .191 \\
\hline MI & $22(48 \%)$ & $29(63 \%)$ & .148 \\
\hline GI bleed & $8(17 \%)$ & $11(23 \%)$ & .477 \\
\hline Remote smoker & $23(50 \%)$ & $33(71 \%)$ & .039 \\
\hline Current smoker & $3(7 \%)$ & $1(2 \%)$ & .240 \\
\hline Previous Sternotomy & $17(37 \%)$ & $24(52 \%)$ & .148 \\
\hline Creatinine & $122(87)$ & $126(70)$ & .809 \\
\hline Hemoglobin & $121(15)$ & $116(13)$ & .091 \\
\hline
\end{tabular}

Values expressed as mean (standard deviation) or number (\%). TAVI, Transapical aortic valve implantation; $B S A$, body surface area; $C C S$, Canadian Cardiovascular Society; $C O P D$, chronic obstructive pulmonary disease; $C V A$, cerebrovascular accident; $H T N$, hypertension; $L V E F$, left ventricular ejection fraction; $M I$, myocardial infarction; NYHA, New York Heart Association classification; $P C I$, percutaneous coronary intervention; $P H T N$, pulmonary hypertension; $P V D$, peripheral vascular disease; GI, gastrointestinal.

valve disease. Generally speaking, transapical AVI is not offered to patients with purely rheumatic aortic stenosis, pure aortic insufficiency, endocarditis or active infection, or severe mitral stenosis. Furthermore, patients with uncorrectable, severe 3-vessel coronary artery disease may not tolerate rapid ventricular pacing during the procedure.

Between the 2 matched groups in this study, there was a trend toward increased 30-day mortality in the transapical AVI group, although this was not statistically significant. However, when analyzing this outcome, it is important to consider that transapical AVI is an emerging approach with limited experience, whereas conventional AVR is a well-established operation. As we have previously demonstrated, there is a significant learning curve associated with the new, evolving transapical procedure. ${ }^{14}$ Our center was the first in the world to perform transapical AVI and thus was not mentored by world experts. The 30-day mortality rate among our first 15 transapical cases was $33 \%$ and significantly decreased to $13 \%$ in subsequent cases, ${ }^{14}$ 
TABLE 5. Preoperative characteristics for matched and unmatched conventional aortic valve replacement cases

\begin{tabular}{lccr}
\hline & $\begin{array}{c}\text { Matched AVR } \\
(\mathbf{n}=\mathbf{4 6})\end{array}$ & $\begin{array}{c}\text { Unmatched AVR } \\
(\mathbf{n}=\mathbf{2 0 4 2})\end{array}$ & $\boldsymbol{P}$ value \\
\hline Year of surgery & $2001-2009$ & $2001-2009$ & \\
Age (y) & $78(6)$ & $69(12)$ & $<.001$ \\
Male & $19(41 \%)$ & $1160(57 \%)$ & .030 \\
BSA & $1.72(0.24)$ & $1.92(0.26$ & $<.001$ \\
NYHA III/IV & $43(93 \%)$ & $821(2 \%)$ & $<.001$ \\
CCS III/IV & $16(35 \%)$ & $387(0.8 \%)$ & $<.001$ \\
LVEF $<35 \%$ & $6(13 \%)$ & $114(6 \%)$ & $<.001$ \\
HTN & $38(83 \%)$ & $1321(65 \%)$ & .011 \\
PHTN & $29(63 \%)$ & $273(13 \%)$ & $<.001$ \\
Dyslipidemia & $32(69 \%)$ & $953(47 \%)$ & .003 \\
PVD & $25(54 \%)$ & $192(9 \%)$ & $<.001$ \\
Arrhythmia & $18(39 \%)$ & $259(13 \%)$ & $<.001$ \\
COPD & $18(39 \%)$ & $389(19 \%)$ & $<.001$ \\
CVA & $15(33 \%)$ & $206(10 \%)$ & $<.001$ \\
Renal dysfunction & $19(41 \%)$ & $291(14 \%)$ & $<.001$ \\
Diabetes & $14(30 \%)$ & $390(19 \%)$ & .062 \\
Liver disease & $5(11 \%)$ & $158(8 \%)$ & .453 \\
Malignancy & $5(11 \%)$ & $296(15 \%)$ & .445 \\
Anemia & $31(67 \%)$ & $200(10 \%)$ & $<.001$ \\
PCI & $11(24 \%)$ & $110(5 \%)$ & $<.001$ \\
MI & $19(41 \%)$ & $186(9 \%)$ & $<.001$ \\
GI bleed & $8(17 \%)$ & $138(7 \%)$ & .009 \\
Remote smoker & $19(41 \%)$ & $530(26 \%)$ & .022 \\
Current smoker & $3(7 \%)$ & $155(8 \%)$ & .800 \\
Previous Sternotomy & $17(37 \%)$ & $96(5 \%)$ & $<.001$ \\
Creatinine & $118(105)$ & $94(53)$ & .129 \\
Hemoglobin & $116(15)$ & $134(16)$ & $<.001$ \\
\hline
\end{tabular}

Values expressed as mean (standard deviation) or number (\%). AVR, Aortic valve replacement; $B S A$, body surface area; $C C S$, Canadian Cardiovascular Society; $C O P D$, chronic obstructive pulmonary disease; $C V A$, cerebrovascular accident; $H T N$, hypertension; $L V E F$, left ventricular ejection fraction; $M I$, myocardial infarction; NYHA, New York Heart Association classification; $P C I$, percutaneous coronary intervention; $P H T N$, pulmonary hypertension; $P V D$, peripheral vascular disease; $G I$, gastrointestinal.

with a current 30 -day mortality rate less than $10 \%$. During our matching process, we included every patient who underwent transapical AVI before May 2010. Thus, we are comparing a new procedure with a well-established approach, which likely underestimates the true benefits of the new procedure once the initial learning curve is overcome. In this study, we saw that $50 \%$ of the perioperative mortalities

TABLE 6. Early clinical outcomes for matched transapical aortic valve implantation and conventional aortic valve replacement cases

\begin{tabular}{lccc}
\hline & TAVI $(\mathbf{n = 4 6 )}$ & AVR $(\mathbf{n}=\mathbf{4 6})$ & $\boldsymbol{P}$ value \\
\hline 30-d mortality & $6(13 \%)$ & $4(9 \%)$ & .527 \\
CVA & $0(0 \%)$ & $2(4 \%)$ & - \\
Wound Infection & $0(0 \%)$ & $0(0 \%)$ & - \\
Reoperation for bleeding & $5(11 \%)$ & $2(4 \%)$ & .629 \\
Postoperative LOS (d) & $9(7)$ & $8(7)$ & .205 \\
\hline
\end{tabular}

Number of patients (\%) or median (interquartile range). TAVI, Transapical aortic valve implantation; $A V R$, aortic valve replacement; $C V A$, Cerebrovascular accident; $L O S$, length of stay.
TABLE 7. Cause of 30-day mortality for matched and unmatched transapical aortic valve implantation cases

\begin{tabular}{|c|c|c|c|}
\hline Sex & Age, $y$ & $\begin{array}{c}\text { Days } \\
\text { postoperative }\end{array}$ & Cause of death \\
\hline
\end{tabular}

Matched TAVI

$\begin{array}{lllll}1 & \text { M } & 91 & 9 & \text { Pneumonia, sepsis, multiorgan failure }\end{array}$

2 M $76 \quad 27 \quad$ Pneumonia, sepsis, multiorgan failure

3 M $91 \quad 11 \quad$ Aspiration pneumonia

$\begin{array}{lllll}4 & \mathrm{M} & 87 & 11 & \text { Ischemic bowel }\end{array}$

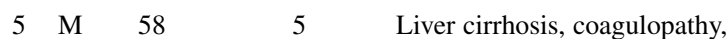
cardiopulmonary arrest, large hemothorax post-chest tube insertion $\begin{array}{llll}6 & \mathrm{~F} & 78 & \text { Intraoperative } \\ \text { Cardiopulmonary arrest before valve }\end{array}$ deployment, heart failure

Unmatched TAVI

$\begin{array}{lllll}1 & F & 71 & 10 & \text { Ischemic bowel }\end{array}$

2 F 82 Intraoperative Left coronary ostia obstruction by native valve leaflet

$\begin{array}{lllll}3 & \text { M } & 78 & 3 & \text { Large pulmonary embolus }\end{array}$

$\begin{array}{lllll}4 & \text { F } & 80 & 5 & \text { Sepsis, multiorgan failure }\end{array}$

$\begin{array}{lllll}5 & \mathrm{M} & 87 & 5 & \text { Cardiopulmonary arrest }\end{array}$

TAVI, Transapical aortic valve implantation.

in the matched transapical AVI group were among the first 15 transapical AVI cases performed at our center. We anticipate that there will be less of a learning curve in new transapical programs, because mentoring can now be provided by experienced, well-qualified surgeons.

Detailed exploration of causes of perioperative mortality provides insight into potential areas of future improvement. Among the patients undergoing transapical AVI, the majority of deaths resulted from infection, multiorgan failure, and exacerbation of preoperative comorbidities, including PVD, liver cirrhosis, and renal dysfunction. This emphasizes the importance of preoperative optimization of patients' other medical conditions, intensive perioperative care, and early, aggressive treatment of complications.

\section{Study Limitations}

Limitations of this study result mainly from the propensity score model. First, as alluded to above, unmeasured or unknown risk factors cannot be included in the propensity score, although they would be accounted for if patients

TABLE 8. Early clinical outcomes for matched and unmatched transapical aortic valve implantation cases

\begin{tabular}{lccc}
\hline & $\begin{array}{c}\text { Matched TAVI } \\
(\mathbf{n}=\mathbf{4 6})\end{array}$ & $\begin{array}{c}\text { Unmatched TAVI } \\
(\mathbf{n}=\mathbf{4 6})\end{array}$ & $\boldsymbol{P}$ value \\
\hline 30-d mortality & $6(13 \%)$ & $5(11 \%)$ & .064 \\
CVA & $0(0 \%)$ & $2(4 \%)$ & - \\
Wound infection & $0(0 \%)$ & $0(0 \%)$ & - \\
Reoperation for bleeding & $5(11 \%)$ & $7(15 \%)$ & $<.001$ \\
Postoperative LOS (d) & $9(7)$ & $8(7)$ & 1.000 \\
\hline
\end{tabular}

Number of patients (\%) or median (interquartile range). TAVI, Transapical aortic valve implantation; CVA, cerebrovascular accident; $L O S$, length of stay. 
TABLE 9. Early clinical outcomes for matched and unmatched conventional aortic valve replacement cases

\begin{tabular}{lccc}
\hline & $\begin{array}{c}\text { Matched AVR } \\
(\mathbf{n}=\mathbf{4 6})\end{array}$ & $\begin{array}{c}\text { Unmatched AVR } \\
(\mathbf{n}=\mathbf{2 0 4 2})\end{array}$ & $\boldsymbol{P}$ value \\
\hline 30-d mortality & $4(9 \%)$ & $37(2 \%)$ & $<.001$ \\
CVA & $2(4 \%)$ & $26(1 \%)$ & .080 \\
Wound infection & $0(0 \%)$ & $10(0.5 \%)$ & - \\
Reoperation for bleeding & $2(4 \%)$ & $153(7 \%)$ & .443 \\
Postoperative LOS (d) & $8(3)$ & $6(4)$ & 1.000 \\
\hline
\end{tabular}

Number of patients (\%) or median (interquartile range). AVR, Aortic valve replacement; $C V A$, cerebrovascular accident; $L O S$, length of stay.

were randomized to the treatment groups. Several potential risk factors, such as concomitant coronary artery disease, frailty, immobility, end-stage liver disease, and end-stage lung disease, are often the reason to decline patients for conventional AVR. However, many of these variables are not collected in the Cardiac Services BC registry and thus could not be included in the matching process. Second, including too many variables in the propensity score will result in none of the patients being matched, whereas using only a small number of variables will produce results that are not clinically meaningful. Consequently, we included only those variables that were demonstrated to be statistically significant in the decision to select a patient for transapical AVI instead of conventional AVR. Because transapical AVI is offered to extremely high-risk patients who previously would have been denied any surgical intervention, it is not possible to match the highest risk patients in a clinically meaningful way. Despite these limitations, a propensitymatched comparison of outcomes after these 2 aortic valve procedures is the best available technique for analyzing these observational data. However, a randomized clinical trial would provide a more accurate comparison of the clinical outcomes for these 2 groups.

Other limitations of this study include the retrospective review of a prospectively maintained database and low event rates resulting in a study potentially underpowered to detect small differences that may be clinically meaningful. Furthermore, future studies should include a comparison of outcomes beyond the perioperative period.

\section{CONCLUSIONS}

Among patients who could be matched, preliminary results show no difference in 30-day mortality or in-hospital complication rates between propensity-matched transapical AVI and conventional AVR cases. However, the lack of study power and the limitations of propensity score matching need to be considered when interpreting this finding. Future studies should also explore outcomes beyond the perioperative period.

\section{References}

1. Bonow RO, Carabello BA, Chatterjee K, de Leon AC Jr, Faxon DP, Freed MD, et al. ACC/AHA 2006 Practice Guidelines for the Management of Patients With Valvular Heart Disease: Executive Summary. J Am Coll Cardiol. 2006;48: 548-675.

2. Bleiziffer S, Ruge H, Mazzitelli D, Schreiber C, Hutter A, Laborde JC, et al. Results of percutaneous and transapical transcatheter aortic valve implantation performed by a surgical team. Eur J Thorac Cardiovasc Surg. 2009;35:615-20.

3. Bleiziffer S, Ruge H, Mazzitelli D, Hutter A, Opitz A, Bauernschmitt R, et al. Survival after transapical and transfemoral aortic valve implantation: talking about two different patient populations. J Thorac Cardiovasc Surg. 2009;138:1073-80.

4. Fusari M, Alamanni F, Bona V, Muratori M, Salvi L, Parolari A, et al. Transcatheter aortic valve implantation in the operating room: early experience. $J$ Cardiovasc Med. 2009;10:383-93.

5. Himbert D, Descoutures F, Al-Attar N, Iung B, Ducrocq G, Détaint D, et al. Results of transfemoral or transapical aortic valve implantation following a uniform assessment in high-risk patients with aortic stenosis. J Am Coll Cardiol. 2009;54: 303-11.

6. Rodes-Cabau J, Dumont E, De LaRochelliere R, Doyle D, Lemieux J, Bergeron $S$, et al. Feasibility and initial results of percutaneous aortic valve implantation including selection of the transfemoral or transapical approach in patients with severe aortic stenosis. Am J Cardiol. 2008;102:1240-6.

7. Thielmann M, Wendt D, Eggebrecht H, Kahlert P, Massoudy P, Kamler M, et al. Transcatheter aortic valve implantation in patients with very high risk for conventional aortic valve replacement. Ann Thorac Surg. 2009;88:1468-74.

8. Webb JG, Altwegg L, Boone RH, Cheung A, Ye J, Lichtenstein SV, et al. Transcatheter aortic valve implantation: impact on clinical and valve-related outcomes. Circulation. 2009;119:3009-16.

9. Leon MB, Smith CR, Mack M, Miller C, Moses JW, Svensson LG, et al. Transcatheter aortic-valve implantation for aortic stenosis in patients who cannot undergo surgery. N Engl J Med. 2010;363:1597-907.

10. Lichtenstein SV, Cheung A, Ye J, Thompson CR, Carere RG, Pasupati S, et al. Transapical transcatheter aortic valve implantation in humans: initial clinical experience. Circulation. 2006;114:591-6.

11. Gutiérrez M, Rodés-Cabau J, Bagur R, Doyle D, DeLarochellière R, Bergeron S, et al. Electrocardiographic changes and clinical outcomes after transapical aortic valve implantation. Am Heart J. 2009;158:302-8.

12. Svensson LG, Dewey T, Kapadia S, Roselli EE, Stewart A, Williams M, et al. United States feasibility study of transcatheter insertion of a stented aortic valve by the left ventricular apex. Ann Thorac Surg. 2008;86:46-54.

13. Walther T, Schuler G, Borger MA, Kempfert J, Seeburger J, Ruckert Y, et al. Transapical aortic valve implantation in 100 consecutive patients: comparison to propensity matched conventional aortic valve replacement. Eur Heart J. 2010;31:1398-403.

14. Ye J, Cheung A, Lichtenstein SV, Altwegg LA, Wong DR, Carere RG, et al. Transapical transcatheter aortic valve implantation: 1-year outcome in 26 patients. J Thorac Cardiovasc Surg. 2009;137:167-73.

15. Ye J, Cheung A, Lichtenstein SV, Nietlispach F, Albugami S, Masson JB, et al. Transapical transcatheter aortic valve implantation: follow-up to 3 years. $J$ Thorac Cardiovasc Surg. 2010;139:1107-13. 